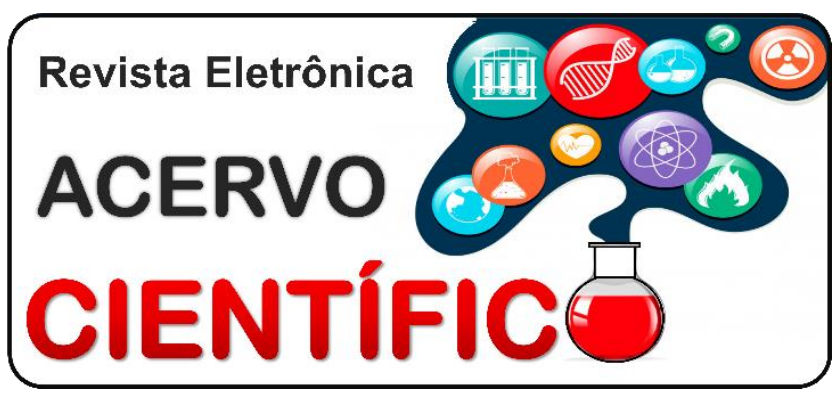

REVISÃO BIBLIOGRÁFICA

Recebido em: 10/2020

Aceito em: $11 / 2020$

Publicado em: 12/2020

\title{
Vantagens da cirurgia do controle de danos comparada aos métodos tradicionais de abordagem ao paciente politraumatizado
}

\author{
Advantages of damage control surgery concerning traditional methods in the care of \\ polytraumatized patients
}

Ventajas de la cirugía de control de daños en comparación a los métodos tradicionales de abordaje al paciente politraumatizado

Layra Ton $^{1 *}$, Wagner Pablo Corrêa ${ }^{2}$, Bernardo Costa Berriel Abreu ${ }^{3}$, Brenda Barreto dos Santos ${ }^{4}$ Daniela Batista Souza ${ }^{2}$, Giovanna Costa Moura Velho ${ }^{5}$, Isabela Cardoso de Araujo ${ }^{3}$, Isabela Oliveira Eugenio ${ }^{2}$, João Pedro Miguez Pinto ${ }^{6}$, Natália Parreira Arantes ${ }^{7}$.

\begin{abstract}
Resumo: Este trabalho relatou as alterações fisiológicas e metabólicas graves que são comumente observadas ao tratar pacientes politraumatizados, as quais culminam na tríade letal do trauma, isto é, coagulopatia, acidose metabólica e hipotermia. Nesse contexto, foram abordados os procedimentos definitivos, ou seja, cirurgia definitiva segura, reconstrução e reabilitação. E os temporários, isto é, intervenções radiológicas, enxerto de stents e o controle de danos. Com isso, ter o êxito de cessar os acometimentos relacionados ao indivíduo politraumatizado, como a tríade letal. Buscou-se, também, evidenciar a importância e os benefícios da utilização do controle de danos em pacientes politraumatizados, cuja técnica é considerada uma inovação significativa em relação aos cuidados desses pacientes, apresentando as situações em que essa abordagem é mais indicada. Esta revisão tem o objetivo de expor as possíveis complicações que podem ser evitadas pela escolha e utilização correta do controle de danos, propiciando, assim, um melhor prognóstico para o paciente.
\end{abstract}

Palavras-chave: Ferimentos e lesões, Traumatismo múltiplo, Procedimentos cirúrgicos.

\begin{abstract}
This work reported the severe physiological and metabolic changes that are commonly observed when treating polytraumatized patients which often culminate in the lethal triad, I.e., coagulopathy, metabolic acidosis, and hypothermia, in inflammatory response syndrome and compensatory anti-inflammatory response syndrome. In this context, the definitive and temporary procedures were addressed, whose objective is the cessation of the involvements related to the polytraumatized individual, such as the lethal triad and syndromes. We sought to highlight the importance and benefits of the use damage control in patients with multiple traumas,
\end{abstract}

\footnotetext{
${ }^{2}$ Instituto Metropolitano de Ensino Superior (IMES), Ipatinga - MG.

${ }^{3}$ Centro Universitário de Volta Redonda (UniFOA), Volta Redonda - RJ.

${ }^{4}$ Faculdade Estácio Alagoinhas (FEA), Alagoinhas - BA.

${ }^{5}$ Centro Universitário de Brasília (UniCEUB), Brasília - DF.

6 Universidade Salvador (UNIFACS), Salvador - BA.

7 Universidade de Vassouras (UV), Vassouras - RJ.
}

${ }^{1}$ Centro Universitário de Caratinga (UNEC), Caratinga - MG. *E-mail: tonlayra@hotmail.com 
whose technique is considered a significant innovation about the care of these patients, presenting the situation in which this method is most indicated. This reveals exposes the recurrences of trauma and the possible complications that can be avoided by the correct use of damage control, providing a better prognosis.

Keywords: Wounds and injuries, Multiple trauma, Surgical procedures.

Resumen: Este estudio informó de los cambios fisiológicos y metabólicos graves que se observan comúnmente al tratar a pacientes politraumatizados, que culminan en la tríada letal de trauma, es decir, coagulopatía, acidosis metabólica e hipotermia. En este contexto, se abordaron los procedimientos definitivos, es decir, la cirugía definitiva segura, la reconstrucción y la rehabilitación. Y las temporales, es decir, las intervenciones radiológicas, el injerto destents y el control de daños. Con esto, para tener éxito en cesar las implicaciones relacionadas con el individuo politraumatizado, como la tríada letal. También buscamos destacar la importancia y los beneficios del uso del control de daños en pacientes politraumatizados, cuya técnica se considera una innovación significativa en relación con el cuidado de estos pacientes, presentando las situaciones en las que este enfoque está más indicado. Esta revisión tiene como objetivo exponer las posibles complicaciones que se pueden evitar eligiendo y utilizando el uso correcto del control de daños, proporcionando así un mejor pronóstico para el paciente.

Palabras clave: Heridas y traumatismos, Traumatismo múltiple, Procedimientos quirúrgicos.

\section{INTRODUÇÃO}

Alterações fisiológicas e metabólicas graves são comumente observadas ao tratar pacientes politraumatizados, e estas podem desencadear a tríade letal, isto é, coagulopatia, acidose metabólica e hipotermia. A partir disso, para estabilizar o paciente e interromper o ciclo da tríade, têm-se os procedimentos definitivos e os procedimentos estabilizadores (PIMENTEL SK, et al., 2017).

Pode-se haver a realização do atendimento total precoce, o qual necessita, em muitos casos, do prosseguimento por um especialista, ou seja, somente um cirurgião experiente seria capaz de realizar tais procedimentos, e nem sempre essa disponibilidade existe no serviço (MACLEAN W, et al., 2019). Além disso, a execução da técnica de cirurgia definitiva implica na tentativa de tratar todas as lesões presentes no paciente simultaneamente, num período de 24 a 48 horas após ocorrer a lesão, sem haver uma etapa anterior a esta que vise deixar o paciente em condições estáveis.

No entanto, apesar dessa intervenção mostrar-se eficiente quando usada em pacientes estáveis, nota-se que ela contribui incisivamente para o aumento da morbidade e da mortalidade de pacientes instáveis, principalmente por conta da Síndrome da Resposta Inflamatória Sistêmica, a Síndrome do Desconforto Respiratório Agudo e a lesão pulmonar aguda (BATES P, 2016; PIMENTEL SK, et al., 2017; GENGFEN HMD, et al., 2014).

Embora 0 atendimento total precoce seja a maneira mais tradicional de tratar os pacientes politraumatizados estáveis, têm-se, também, os procedimentos denominados estabilizadores ou temporários, ou seja, procedimentos que visam a correção hemodinâmica e respiratória do paciente antes de corrigir as demais lesões, como as do sistema locomotor, e eles vêm ganhando crescente notoriedade no âmbito cirúrgico, a saber, intervenções radiológicas para embolizações seletivas, utilização de stents em lesões vasculares periféricas maiores e a cirurgia de controle de danos (CD) (NICOLA R, 2013; ARYA AW, 2018).O $C D$ é uma abordagem que vem ganhando espaço no cuidado do paciente politraumatizado, sendo um procedimento estabilizador, o qual tem se desenvolvido desde o último século (ROBERTS DJ, et al., 2016).

Trata-se de uma estratégia dinâmica, estruturada e adaptável, que pode ser utilizada até mesmo no atendimento pré-hospitalar, tendo sua origem inclusive pautada neste cenário, em que em situações nas quais se haja a impossibilidade de garantir ao politraumatizado o acesso direto imediato a um centro de saúde, como um hospital, é realizado o CD de forma a estabilizar o paciente até que ele chegue no local em seja 
possível realizar o tratamento resolutivo necessário (POSADA MAG, et al., 2019). O propósito do CD é diminuir o risco de o paciente vir a óbito através da estabilização prévia do quadro do paciente antes que sejam realizados os procedimentos resolutivos. A execução do $C D$ utiliza-se de dois procedimentos, a cirurgia de $C D$ e a ressuscitação de controle de danos (RCD), de forma que se utiliza inicialmente a RCD para, primeiramente, atingir a hemostasia e afastar o paciente da tríade letal, e, posteriormente, utiliza-se a cirurgia de CD com o propósito de se focar na reconstrução anatômica e na descontaminação das áreas injuriadas. Assim, dentre as vantagens do CD, pode-se citar a diminuição do tempo de cirurgia, prevenção de danos fisiológicos irreversíveis e favorecimento à recuperação no centro de terapia intensiva (ROBERTS DJ, et al., 2017; MACLEAN W, et al., 2019).

Quando o cirurgião possui capacitação adequada em relação à cirurgia de CD e sua indicação, a realização do CD reduz o índice de mortalidade dos pacientes (PIMENTEL SK, et al., 2017). Entretanto, quando indicado de maneira equívoca, o CD se associar ao aumento da morbidade e de gasto desnecessário dos recursos hospitalares. Um estudo revelou que o procedimento controle de danos foi realizado de maneira adequada em apenas $80 \%$ dos pacientes, ou seja, ainda é preciso aprimorar a capacitação dos profissionais em relação à escolha da intervenção mais adequada (BENZ D e BALOGH ZJ, 2017). Assim, denota-se a importância da realização correta do $C D$, uma vez que é um procedimento o qual diminui a probabilidade de óbito e propicia maior estabilidade ao paciente antes da realização dos procedimentos definitivos (PIMENTEL SK, et al., 2017).

A presente revisão tem como objetivo demonstrar em que situação é mais indicado fazer o CD e não prosseguir diretamente para o atendimento total precoce, uma vez que a escolha correta do procedimento é determinante para preservar a vida do paciente em estado crítico.

\section{REVISÃO BIBLIOGRÁFICA}

A presença de múltiplas injúrias simultaneamente em um paciente vítima de trauma caracteriza o politraumatismo sabe-se que a escolha da técnica cirúrgica e da terapêutica correta é essencial para um bom prognóstico deste paciente. Assim, têm-se algumas maneiras de manusear o paciente politraumatizado, minimizando os danos e a exaustão fisiológica. Dessa forma, houve uma mudança no tratamento do paciente politraumatizado, que por muito tempo, foi centralizado no bloco cirúrgico, de maneira que a estratégia era corrigir todas as lesões do paciente. No entanto, há um método que se destaca com notoriedade no âmbito do cuidado do indivíduo politraumatizado, o controle de danos (ROBERTS DJ, et al., 2017).

Nesse contexto, o CD é uma estratégia usada em que o tratamento se baseia no cessamento de quadros hemorrágicos exsanguinante e de contaminação grave em pacientes com distúrbios fisiológicos ou com um padrão de lesões de alto risco. Ademais, $O C D$ baseia-se em uma técnica cirúrgica e ressuscitação com controle de danos a fim de evitar o desencadeamento da tríade letal e outras morbidades que o paciente que sofreu múltiplas injurias pode apresentar. Assim, após o ato cirúrgico, o paciente é internado na unidade de terapia intensiva (UTI), onde ocorre uma reanimação contínua, que consiste em rápido controle do quadro hemorrágico, hipotensão permissiva, uso reduzido de cristaloides e administração de hemoderivados em quantidade próxima ao volume sanguíneo (ROBERTS DJ, et al., 2017).

O paciente politraumatizado pode evoluir para uma cascata de eventos caracterizada como a tríade letal ou a tríade da morte, a qual é composta por três fatores organizacionais predeterminantes na sobrevivência do paciente politraumatizado, sendo eles: hipotermia, coagulopatia e acidose metabólica. Além disso, o prognóstico do doente será definido por meio desses preditores (MUTHUKUMAR V, et al., 2019).

Dessa forma, tem-se a hipotermia, que é caracterizada pela queda da temperatura corpórea e é um importante agravante no quadro clínico da vítima de trauma, visto que quanto menor a temperatura, maior a probabilidade de mortalidade do politraumatizado. Temperaturas abaixo de $35^{\circ} \mathrm{C}$ revelam pior prognóstico e há evidências de $100 \%$ de mortalidade quando a queda se estende a 32으 (EDELMUTH RCL, et al., 2013). Ainda é importante salientar que a hipotermia é um pró fator desencadeador de arritmias ventriculares que ocasionam diminuição da pós carga e, assim, aumenta a resistência vascular periférica, ocasionando um desarranjo sistêmico (EDELMUTH RCL, et al., 2013). 
A hipotermia é um importante desencadeador no distúrbio de coagulação, pois inibe a interação do fator de Von Willebrand com as glicoproteínas plaquetárias, colaborando para uma disfunção plaquetária também inativa os fatores de coagulação dependentes da temperatura corpórea, ocasionando alterações no sistema fibrinolítico e gerando anormalidades endoteliais. Assim, quando o paciente politraumatizado atinge temperaturas menores que $35^{\circ} \mathrm{C}$, há maior probabilidade de piora de seu quadro clínico (EDELMUTH RCL, et al., 2013). Tem-se, ainda, a perfusão tissular prejudicada, a qual também está associada à coagulopatia resultante da anticoagulação endógena precoce e da fibrinólise (MUTHUKUMAR V, et al., 2019).

É comum que haja intensa perda do volume sanguíneo em vítimas de múltiplas injúrias, o que ativa o mecanismo de vasoconstrição periférica de forma compensatória, o qual é controlado devido a uma intensa carga simpática, acarretando uma hipoperfusão tecidual na tentativa de regular o volume corporal. Diante a isso, ocorre a redução na oferta de oxigênio e a mudança do metabolismo aeróbico em anaeróbico, fator determinante para surgimento da acidose metabólica (EDELMUTH RCL, et al., 2013).

A queda no $\mathrm{pH}$ interno também é um fator significante para estabelecer o prognóstico do paciente politraumatizado, uma vez que o $\mathrm{pH}$ que se mantém abaixo de 7,2 por tempo prolongado sinaliza uma má evolução do doente, e é diretamente relacionado com o alto índice de mortalidade. Além disso, há uma sobrecarga do sistema respiratório na tentativa de reverter o desequilíbrio metabólico em que o organismo se encontra, portanto, todas essas alterações podem culminar em diminuição de contratilidade do miocárdio e redução da pós-carga (EDELMUTH RCL, et al., 2013).

Sendo assim, é importante que a cirurgia de controle de dano seja realizada com excelência, pois a sua não realização pode a acarretar consequências devastadoras, como ativação da tríade letal e falência múltipla de órgãos, levando o paciente ao óbito (MALGRAS B, et al., 2017).

A partir disso, tem-se a cirurgia definitiva segura, que é apresentada como uma associação ativa dos métodos de atendimento total precoce de controle de danos e ortopedia de controle de danos. Ademais, os procedimentos definitivos envolvem, também, reconstrução e reabilitação. Então, quando as lesões restantes e reparações são finalmente realizadas, é proporcionada ao paciente a recuperação de funcionalidade e, consequentemente, a sua volta ao mercado de trabalho (PLEIFER R e PAPE HC, 2016; MALGRAS B, et al., 2017).

Ademais, os pacientes são classificados em quatro grupos diferentes, nomeados como estável, limítrofe ou borderline, instável e in extremis. Os pacientes estáveis respondem à terapia inicial de volume e são capazes de realizar a cirurgia definitiva segura, o grupo in extremispossue relevante risco de vida devido a perda descontrolada sanguínea, esses são encaminhados para UTI para monitoramento e melhor suporte, o grupo classificado como limítrofe possui episódios instáveis cardiopulmonares e o grupo de instáveis dispõe de instabilidade cardiopulmonar constantemente, diante disso os estágios limítrofe e instável são levados para UTI para reanimação e, posteriormente, são reavaliados em relação à sua estabilidade para que seja realizada a cirurgia definitiva segura (PLEIFER R e PAPE HC, 2016; KLUTER T, et al., 2013).

Além disso, estudos concluíram que o método de atendimento total precoce é inaceitável para todas as vítimas de fraturas traumáticas, pois em situações que há instabilidade e complicações associadas, esse procedimento foi relacionado a um elevado número de complicações pós-operatórias graves, como a Síndrome da Resposta Inflamatória Sistémica, a Síndrome do Desconforto Respiratório Agudo e a lesão pulmonar aguda (GENGFEN HMD, et al., 2014).

Em situações que a prioridade são os procedimentos que salvam vidas a ortopedia de controle de danos torna-se o melhor método, um estudo prospectivo randomizado publicado no final dos anos 80 conceituou o cuidado total precoce (CTP) e evidenciou um declínio da morbimortalidade através da estabilização precoce de lesões de ossos longos em pacientes politraumatizados (BATES P, 2016).

O CTP envolve a fase inicial do tratamento, em um período de 24 a 48 horas, em que ocorre a fixação cirúrgica definitiva da fratura. A estabilização operatória dessas fraturas demonstrou diminuir os agravos pulmonares, o tempo na UTI e a internação hospitalar em comparação ao tratamento tradicional de fratura não operatória (HAN G, et al., 2014; NICOLA R, 2013). 
Visões contraditórias surgiram a partir da década de 90 (NICOLA R, 2013). O CTP não foi considerado adequado para todos os politraumatizados, uma vez que ocorreu uma alta associação complicações pulmonares de maneira inesperada em pacientes instáveis, como síndrome da resposta inflamatória sistêmica (SIRS), lesão pulmonar aguda (LPA), síndrome do desconforto respiratório agudo (SDRA) e síndrome de disfunção de múltiplos órgãos (SDMO) (HAN G, et al., 2014; BATES P, 2016).

Os procedimentos de fechamento temporário da cavidade abdominal são a melhor forma de facilitar reoperações cirúrgicas quando necessárias. Entretanto, apesar desse tipo de procedimento ser muito benéfico, há algumas consequências negativas para o paciente, por exemplo, a retração lateral da aponeurose durante a permanência do abdome aberto, o que dificulta o fechamento da cavidade abdominal e pode ocasionar o surgimento de hérnias incisionais.

Consequentemente, o procedimento pode levar à formação de bridas e tornar cirurgias futuras mais complexas com um aumento da mortalidade; contudo, elas podem ser evitadas empregando-se algumas técnicas diferentes, como Vaccum-pack e Vaccum-Assisted ClosureTherapy (VAC), as quais conseguem diminuir a tensão do fechamento da cavidade abdominal e, dessa forma, amenizar as complicações por hérnias, a quantidade de citocinas pró-inflamatórias e a infecção bacteriana (RIBEIRO JR MAF, et al., 2016; SALAMONE G, et al., 2017).

Além disso, o conceito de tratamento do paciente politraumatizado, por muito tempo, era centralizado no bloco cirúrgico, de maneira que a estratégia era corrigir todas as lesões do paciente. No entanto, um novo método tem ganhado notoriedade no âmbito do cuidado do indivíduo politraumatizado, o CD. Nesse contexto, o CD é uma nova estratégia de controle de danos, na qual o tratamento baseia-se no cessamento de quadros hemorrágicos exsanguinante e de contaminação grave em pacientes com distúrbios fisiológicos ou com um padrão de lesões de alto risco.

Ademais, o CD baseia-se em uma técnica cirúrgica e ressuscitação com controle de danos. Assim, após o ato cirúrgico, o paciente é internado na UTI, onde ocorre uma reanimação contínua, que consiste em rápido controle do quadro hemorrágico, hipotensão permissiva, uso reduzido de cristaloides e administração de hemoderivados em quantidade próxima ao volume sanguíneo (ROBERTS DJ, et al., 2017).

A cirurgia de controle de danos é utilizada para realizar a manutenção do abdome aberto. Assim, trata-se o paciente para estabilizá-lo hemodinamicamente, prioritariamente, postergando alguns procedimentos, os quais poderiam agravar ainda mais os parâmetros da hemodinâmica do paciente (RIBEIRO JR, et al., 2016).

Ainda, é importante lembrar que quase $25 \%$ dos pacientes politraumatizados apresentam uma coagulopatia induzida pelo trauma, caracterizada por uma hemorragia inicial somada a fatores prócoagulantes atrasados. Portanto, é imprescindível iniciar o mais rápido possível a conduta de controle de danos após trauma severo e em pacientes com coagulopatia traumática aguda (TONGLET M, 2018).

Ademais, a identificação precoce de pacientes que precisam de procedimentos cirúrgicos temporários possui o potencial de ser benéfico para unidades de emergência que não são especializadas em trauma e que provavelmente não estarão sempre preparadas para receberem casos assim. Por outro lado, essa identificação também pode ser útil em centros de alto desempenho de trauma, para analisar quais pacientes devem ser tratados prioritariamente (TONGLET M, 2018).

As principais indicações para a realização do controle de danos são: incapacidade de se fechar a cavidade abdominal, infecção grave, e consequente necessidade de drenagem da cavidade abdominal, hipertensão intra-abdominal documentada, síndrome de compartimento abdominal e tríade da morte (Figura 1) (RIBEIRO JR MAF, et al., 2016).

Além disso, observou-se que a cirurgia para controle de danos se mostrou eficiente, também, em quadros não traumáticos, principalmente, em pacientes com isquemia mesentérica aguda, instabilidade hemodinâmica decorrente de sepse grave, choque hipovolêmico hemorrágico e infecções necrotizantes (EDELMUTH RCL, et al., 2013). 
Figura 1 - Principais indicações para realização do controle de danos.

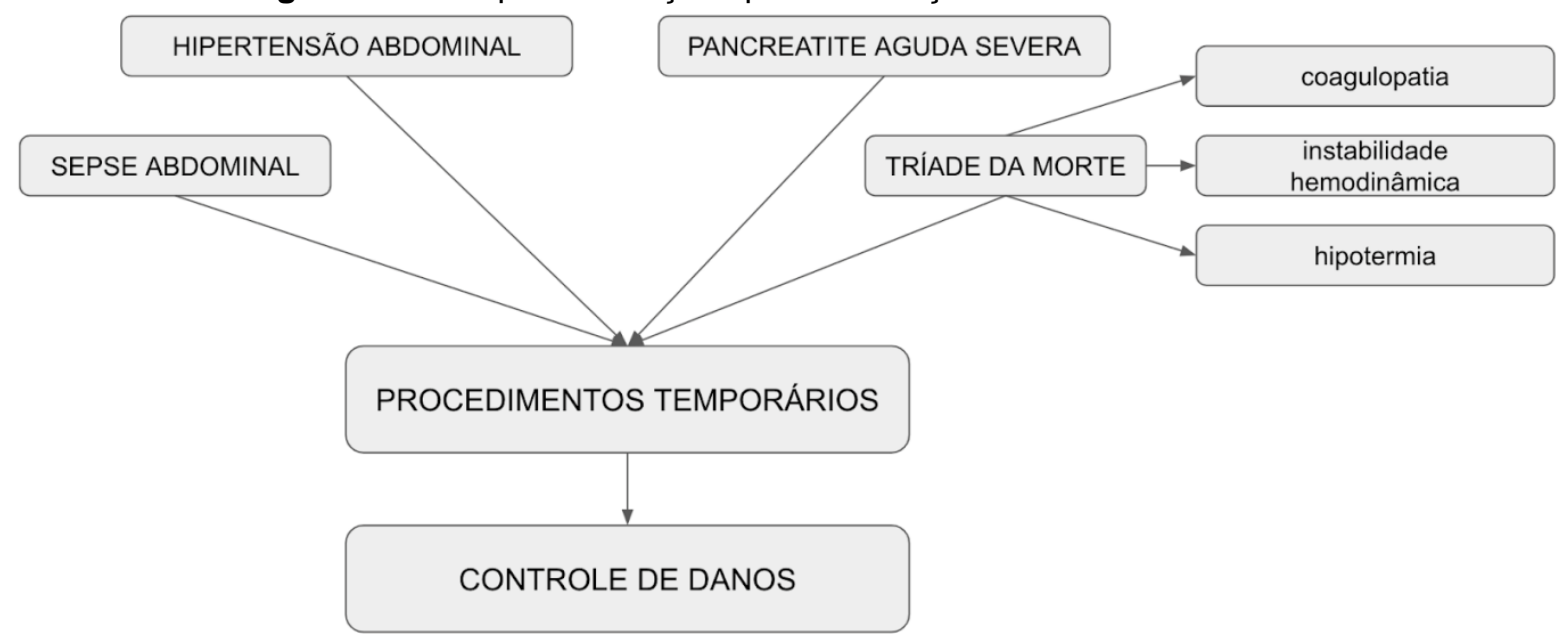

Fonte: TON L, et al., 2020; Adaptado de: SALOMONE G, 2018.

Nesse contexto, a principal finalidade do $C D$ em cenários agudos é realizar o menor número de intervenções para evitar ainda mais danos. A título de exemplificação, em meados de 2011, estudos relataram que os parâmetros ácido-base e dosagem de lactato poderiam ajudar na escolha do melhor método de abordagem do paciente politraumatizado, ou seja, se o indivíduo possuir valores aumentados de bases e lactatos, os quais possam ser normalizados, é preferível poupar o método CD (GUERADO E, et al., 2019).

Sabe-se que existem duas opções de tratamento para pacientes com fraturas traumáticas em nosso cotidiano: o procedimento de CTP e o CD (HAN G, et al., 2014). No entanto, debates atuais têm ocasionado discussões sobre a relevância de vários marcadores laboratoriais (GUERADO E, et al., 2019). Marcadores, mediadores e células efetoras da resposta inflamatória sistêmica se acentuaram por meio da cirurgia de fratura definitiva, a interleucina-6 (IL-6) singularmente foi correlacionada com a SIRS (BATES P, 2016; WEINBERG D, et al., 2015).

O achado que a cirurgia de fratura definitiva estagiada, após fixação externa temporária, não foi associada à nova elevação de IL-6 corroborou a abordagem ortopédica do CD, pacientes politraumatizados que receberam CTP apresentaram uma taxa elevada de complicações, como SDRA, SIRS e SDMO, as quais são espectros da mesma condição fisiológica (HAN G, et al., 2014; BATES P, 2016).

O tratamento por meio do CD produz baixas taxas de incidência de LPA e SDRA em relação ao CTP. Constata-se, também, que pacientes tratados por meio do $C D$ exibiram menores complicações no pósoperatório em relação a aqueles que foram tratados com CTP, ratificando a eficácia do CD durante a fase aguda de ressuscitação para fraturas pélvicas limítrofes (HAN G, et al., 2014; NICOLA R, 2013).

\section{CONSIDERAÇÕES FINAIS}

A técnica de $C D$, na maioria dos casos, mostrou-se eficaz em comparação às técnicas tradicionais quando aplicada corretamente. Esse método proporciona uma melhor resposta pós-operatória e, consequentemente, um melhor prognóstico aos politraumatizados. Contudo, algumas variáveis, como o tipo de trauma além da experiência médica com os procedimentos de controle, devem ser consideradas, com o objetivo de reduzir a morbimortalidade e aumentar a sobrevida desses pacientes após as abordagens cirúrgicas.

\section{REFERÊNCIAS}

1. ARYA AW. Reanimación con control de daños en el trauma grave pediátrico. Revista Chilena de Pediatría, 2018; 89(1): 118-127.

2. BATES P, et al. Demystifyingdamage control in musculoskeletal trauma. Royal College of Surgeons, 2016; 98: 291294. 
3. BENZ D, BALOGH ZJ. Damage control surgery: current state and future directions. Current Opinion in Critical Care, $2017 ; 23(6): 491-497$.

4. EDELMUTH RCL, et al. Cirurgia para controle de danos: estado atual. Revista do Colégio Brasileiro de Cirurgiões, 2013; 40(2).

5. GENGFEN HMD, et al. Damage-Control Orthopedics Versus Early Total Care in the Treatment of Borderline HighEnergy Pelvic Fractures. Feature Article, 2014; 37(12): 1091-1100.

6. GUERADO E, et al. Damage control orthopaedics: State of the art. World J Orthop, 2019; 10(1): 1-13.

7. HAN G, et I. Damage-control orthopedics versus early total care in the treatment of borderline high-energy pelvic fractures. Orthopedics, 2014; 37 (12): e1091-e1100.

8. KLUTER T, et al. Operative Versorgungs-strategion von Polytraumapatienten. Leitthema, 2013; 739-763.

9. MACLEAN W, et al. Trauma laparotomy and damage control surgery. Surgery Journal, 2019; 37: 549-557.

10. MALGRAS B, et al. Damage control: Concept and implementation. Journal of visceral surgery, 2017; 154: S19-S29.

11. MUTHUKUMAR V, et al. Concept of Lethal Triad in Critical Care of Severe Burn Injury. Indian Journal of critical care medicine, 2019; 23(5): 206-209.

12. NICOLA R. Early Total Care versus Damage Control: Current Concepts in the Orthopedic Care of Polytrauma Patients. ISRN Orthopedics, 2013; 2013: 01-09.

13. PIMENTEL SK, et al. Cirurgia de controle de danos: estamos perdendo controle das indicações? Revista do Colégio Brasileiro de Cirurgiões, 2018; 45(1): 01-06.

14. PLEIFER R, PAPE HC. Diagnostique and Versorgungs strategien beimpoly traumatisierten Patienten. Springermedizin. decme, 2016; 1-9.

15. POSADA MAG, et al. Damage Control Resuscitation en el paciente traumático. Revista Española de Anestesiología y Reanimación, 2019; 66 (7): 294-404.

16. RIBEIRO JR, et al. Estudo comparativo de técnicas de fechamento temporário da cavidade abdominal durante 0 controle de danos. Rev. Col. Bras. Cir., 2016; 43 (5): 368-373.

17. ROBERTS DJ, et al. History of the Innovation of Damage Control for Management of Trauma Patients: $1902-2016$. Annals of Surgery, 2016; 265: 1034-1044.

18. SALAMONE G, et al. Vacuum-Assisted Wound Closure with Mesh-Mediated Fascial Traction Achieves Better Outcomes than Vacuum-Assisted Wound Closure Alone: A Comparative Study. World J Surg, $2018 ; 42$ (6): 1679 1686.

19. STINNER DJ, EDWARDS D. Surgical Management of Musculoskeletal. Cross Mark, 2017; 1120-1131.

20. TONGLET M, et al. Impact of a prehospital discrimination between trauma patients with or without early acute coagulopathy of trauma and the need for damage control resuscitation: rationale and design of a multicenter randomized phase II trial. Acta ChirBelg, 2019; 119 (2): 88-94.

21. WEINBERG D, et al. Prolonged resuscitation of metabolic acidosis after trauma is associated with more complications. Journal of Orthopaedic Surgery and Research, 2015; 10-15. 